\title{
Supporting Information: Reactive Force Field Modeling of Zinc Oxide Nanoparticle Formation
}

\author{
Craig J. Tainter and George C. Schatz* \\ Department of Chemistry \\ Northwestern University \\ 2145 Sheridan Road, Evanston, IL, 60208, USA \\ Email: g-schatz@northwestern.edu
}

\section{ReaxFF Parameter File}

Below is the final parameter file used for the MD simulations with LAMMPS.

Reactive MD-force field: C. J. Tainter and G. C. Schatz; Zn,O,C,H

39 ! Number of general parameters

50.0000 !Overcoordination parameter

9.5469 !Overcoordination parameter

26.5405 !Valency angle conjugation parameter

1.7224 !Triple bond stabilisation parameter

6.8702 !Triple bond stabilisation parameter

60.4850 !C2-correction

1.0588 !Undercoordination parameter

4.6000 !Triple bond stabilisation parameter

12.1176 !Undercoordination parameter

13.3056 !Undercoordination parameter

-70.5044 !Triple bond stabilization energy

0.0000 !Lower Taper-radius

10.0000 !Upper Taper-radius

2.8793 !Not used

33.8667 !Valency undercoordination

6.0891 !Valency angle/lone pair parameter

1.0563 !Valency angle

2.0384 !Valency angle parameter

6.1431 !Not used

6.9290 !Double bond/angle parameter

0.3989 !Double bond/angle parameter: overcoord

3.9954 !Double bond/angle parameter: overcoord

-2.4837 !Not used

5.7796 !Torsion/BO parameter

10.0000 !Torsion overcoordination

1.9487 !Torsion overcoordination

-1.2327 !Conjugation 0 (not used)

2.1645 !Conjugation

1.5591 !vdWaals shielding

0.1000 !Cutoff for bond order $(* 100)$

2.1365 !Valency angle conjugation parameter

0.6991 !Overcoordination parameter

50.0000 !Overcoordination parameter 
1.8512 ! Valency/lone pair parameter

0.5000 !Not used

20.0000 !Not used

5.0000 !Molecular energy (not used)

0.0000 !Molecular energy (not used)

2.6962 !Valency angle conjugation parameter

4 ! Nr of atoms ; cov.r ; valency ; a.m ; Rvdw ; Evdw ; gammaEEM ; cov.r2 ; \# alfa ; gammavdW ; valency ; Eunder ; Eover ; chiEEM ; etaEEM ; n.u. cov r3 ; Elp ;Heat inc.; n.u. ; n.u. ; n.u. ; n.u. ;n.u. ov/un ; val1 ; n.u. ; val3 ; vval4 ; n.u. ; n.u.. ; n.u.

$\begin{array}{lllllllll}\mathrm{H} & 0.8930 & 1.0000 & 1.0080 & 1.3550 & 0.0930 & 0.8203 & -0.1000 & 1.0000\end{array}$

$\begin{array}{llllllll}8.2230 & 33.2894 & 1.0000 & 0.0000 & 121.1250 & 3.7248 & 9.6093 & 1.0000\end{array}$

$\begin{array}{llllllll}-0.1000 & 0.0000 & 61.6606 & 3.0408 & 2.4197 & 0.0003 & 1.0698 & 0.0000\end{array}$

$\begin{array}{llllllll}-19.4571 & 4.2733 & 1.0338 & 1.0000 & 2.8793 & 0.0000 & 0.0000 & 0.0000\end{array}$

$\begin{array}{lllllllll}\text { O } & 1.2450 & 2.0000 & 15.9990 & 2.3890 & 0.1000 & 1.0898 & 1.0548 & 6.0000\end{array}$

$\begin{array}{llllllll}9.7300 & 13.8449 & 4.0000 & 37.5000 & 116.0768 & 8.5000 & 8.3122 & 2.0000\end{array}$

$\begin{array}{llllllll}0.9049 & 0.4056 & 59.0626 & 3.5027 & 0.7640 & 0.0021 & 0.9745 & 0.0000\end{array}$

$\begin{array}{llllllll}-3.5500 & 2.9000 & 1.0493 & 4.0000 & 2.9225 & 0.0000 & 0.0000 & 0.0000\end{array}$

$\begin{array}{lllllllll}\text { Zn } & 1.8862 & 2.0000 & 65.3900 & 1.9200 & 0.2998 & 0.4828 & -1.6836 & 2.0000\end{array}$

$\begin{array}{llllllll}11.5134 & 18.3776 & 2.0000 & 0.0078 & 0.0000 & 2.0219 & 5.7915 & 0.0000\end{array}$

$\begin{array}{llllllll}-1.2000 & 0.0000 & 266.4838 & 5.3430 & 10.1260 & 0.7590 & 0.0000 & 0.0000\end{array}$

$\begin{array}{llllllll}-3.0614 & 2.1158 & 1.0338 & 6.2998 & 2.5791 & 0.0000 & 0.0000 & 0.0000\end{array}$

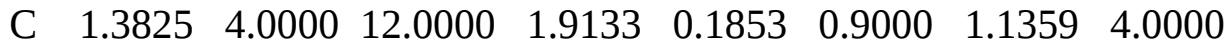

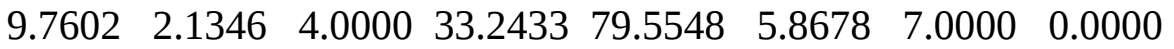

$\begin{array}{lllllllll}1.2104 & 0.0000 & 199.0303 & 8.6991 & 34.7289 & 13.3894 & 0.8563 & 0.0000\end{array}$

$\begin{array}{llllllll}-2.8983 & 2.5000 & 1.0564 & 4.0000 & 2.9663 & 0.0000 & 0.0000 & 0.0000\end{array}$

10 ! Nr of bonds ; Edis1 ; LPpen ; n.u. ; pbe1 ; pbo5 ; 13corr ; pbo6

pbe2 ; pbo3 ; pbo4 ; n.u. ; pbo1 ; pbo2 ; ovcorr

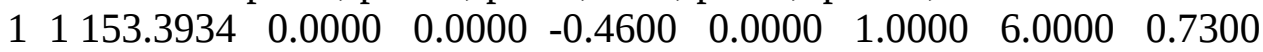

$\begin{array}{llllllll}6.2500 & 1.0000 & 0.0000 & 1.0000 & -0.0790 & 6.0552 & 0.0000 & 0.0000\end{array}$

$\begin{array}{llllllllll}12 & 160.00000 & 0.0000 & 0.0000 & -0.57249999 & 0.0000 & 1.0000 & 6.0000 & 0.5626\end{array}$

$\begin{array}{llllllll}1.1150000 & 1.0000 & 0.0000 & 0.0000 & -9.20000002 \mathrm{E}-02 & 4.2789998 & 0.0000 & 0.0000\end{array}$

$\begin{array}{llllllllll}1 & 3 & 0.0000 & 0.0000 & 0.0000 & 0.0000 & -0.5000 & 1.0000 & 50.0000 & 0.5000\end{array}$

$\begin{array}{llllllll}0.5000 & -0.5000 & 30.0000 & 1.0000 & -0.2000 & 8.0000 & 0.0000 & 0.0000\end{array}$

$\begin{array}{llllllllll}1 & 4 & 169.47600 & 0.0000 & 0.0000 & -0.60829997 & 0.0000 & 1.0000 & 6.0000 & 0.7652\end{array}$

$\begin{array}{llllllll}5.2290001 & 1.0000 & 0.0000 & 1.0000 & -5.00000007 \mathrm{E}-02 & 6.9136000 & 0.0000 & 0.0000\end{array}$

$22142.2858145 .000050 .8293 \quad 0.2506 \quad-0.1000 \quad 1.0000 \quad 29.7503 \quad 0.6051$

$\begin{array}{llllllll}0.3451 & -0.1055 & 9.0000 & 1.0000 & -0.1225 & 5.5000 & 1.0000 & 0.0000\end{array}$

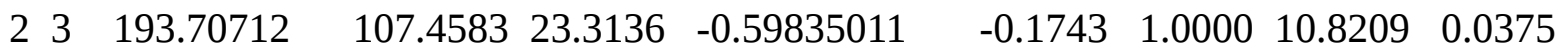

$\begin{array}{llllllll}1.7527413 & -0.3113 & 7.0000 & 1.0000 & -0.34207681 & 5.4933271 & 0.0000 & 0.0000\end{array}$

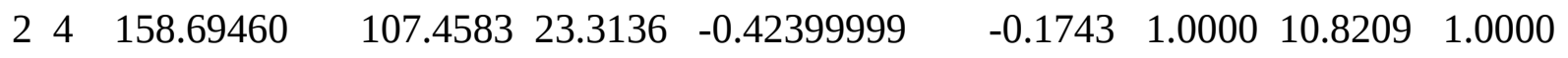

$\begin{array}{llllllll}0.53219998 & -0.3113 & 7.0000 & 1.0000 & -0.14470001 & 5.2449999 & 0.0000 & 0.0000\end{array}$

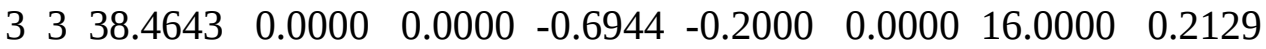

$\begin{array}{llllllll}0.5059 & -0.2000 & 15.0000 & 1.0000 & -0.0814 & 6.0333 & 0.0000 & 0.0000\end{array}$

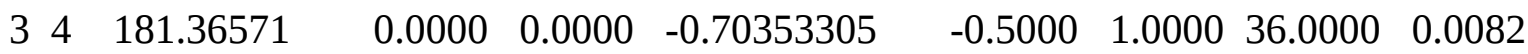

$\begin{array}{llllllll}1.9281975 & -0.2500 & 20.0000 & 1.0000 & -0.34674627 & 4.6113410 & 0.0000 & 0.0000\end{array}$

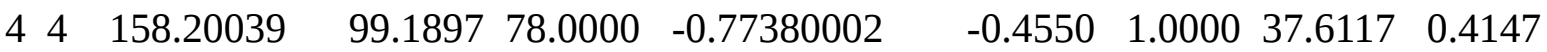

$\begin{array}{llllllll}0.45899999 & -0.1000 & 9.1628 & 1.0000 & -7.76999965 E-02 & 6.7268000 & 1.0000 & 0.0000\end{array}$

6 ! Nr of off-diagonal terms ; Ediss ; Ro ; gamma ; rsigma ; rpi ; rpi2 


$\begin{array}{llllllll}1 & 2 & 0.0283 & 1.2885 & 10.9190 & 0.9215 & -1.0000 & -1.0000 \\ 1 & 3 & 0.0987 & 1.8227 & 12.0654 & 0.1000 & -1.0000 & -1.0000 \\ 1 & 4 & 0.1219 & 1.4000 & 9.8442 & 1.1203 & -1.0000 & -1.0000 \\ 2 & 3 & 0.2744 & 2.1414 & 9.7703 & 1.9804 & -1.0000 & -1.0000 \\ 2 & 4 & 0.1131 & 1.8523 & 9.8442 & 1.2775 & 1.1342 & 1.0621 \\ 3 & 4 & 0.32264534 & 1.8473080 & 10.474574\end{array}$

29 ! Nr of angles ; at1 ; at2 ; at3 ; Thetao ; o ; ka ; kb ; pv1 ; pv2

$44459.057330 .70290 .7606 \quad 0.00000 .7180 \quad 6.29331 .1244$

$44165.775814 .52346 .2481 \quad 0.00000 .5665 \quad 0.00001 .6255$

$14170.260725 .22023 .7312 \quad 0.00000 .0050 \quad 0.00002 .7500$

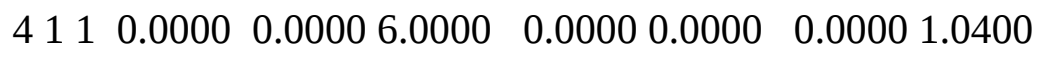

$\begin{array}{lllllll}414 & 0.0000 & 3.41107 .7350 & 0.0000 & 0.0000 & 0.0000 & 1.0400\end{array}$

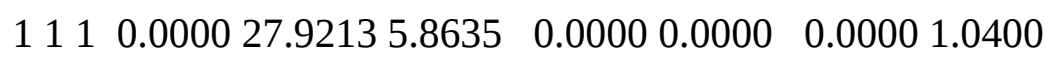

$44249.68117 .17134 .3889 \quad 0.00000 .7171 \quad 10.26611 .0463$

$24277.747340 .17182 .9802-25.30631 .6170-46.13152 .2503$

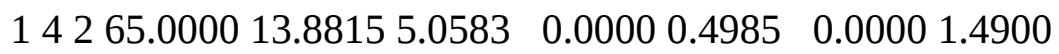

$42473.531244 .72750 .7354 \quad 0.00003 .0000 \quad 0.00001 .0684$

$\begin{array}{lllllll}42279.476136 .3701 & 1.8943 & 0.0000 & 0.7351 & 67.67773 .0000\end{array}$

$22280.732430 .45540 .9953 \quad 0.00001 .631050 .00001 .0783$

$\begin{array}{lllllll}42170.1880 & 20.9562 & 0.3864 & 0.0000 & 0.0050 & 0.0000 & 1.6924\end{array}$

$\begin{array}{llllll}12275.6935 & 50.00002 .0000 & 0.00001 .0000 & 0.0000 & 1.1680\end{array}$

$\begin{array}{llllll}12 & 185.8000 & 9.84532 .2720 & 0.00002 .8635 & 0.0000 & 1.5800\end{array}$

$\begin{array}{lllllll}412 & 0.000025 .00003 .0000 & 0.0000 & 1.0000 & 0.0000 & 1.0400\end{array}$

$\begin{array}{lllllll}212 & 0.0000 & 15.00002 .8900 & 0.0000 & 0.0000 & 0.00002 .8774\end{array}$

$\begin{array}{lllllll}112 & 0.0000 & 8.57443 .0000 & 0.0000 & 0.0000 & 0.0000 & 1.0421\end{array}$

$\begin{array}{lllllll}12 & 377.5446 & 9.90162 .3157 & 0.0000 & 0.4543 & 0.0000 & 2.3770\end{array}$

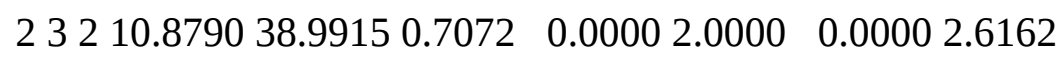

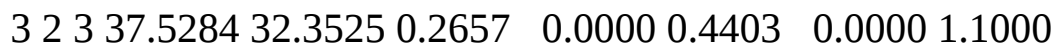

$23316.962430 .32410 .2697 \quad 0.00002 .0000 \quad 0.00003 .0708$

$22360.000020 .00000 .5000 \quad 0.00001 .0000 \quad 0.00002 .0000$

$\begin{array}{llllllll}434 & 9.2000017 & 33.667389 & 0.67785233 & 0.0000 & 4.4482174 & 0.0000 & 1.9950953\end{array}$

$\begin{array}{llllllll}341 & 44.407127 & 5.6261773 & 2.0199990 & 0.0000 & 1.6999993 & 0.0000 & 3.2799988\end{array}$

$\begin{array}{llllllll}344 & 24.772160 & 13.170823 & 4.0599985 & 0.0000 & 1.2895108 & 0.0000 & 1.5386710\end{array}$

$\begin{array}{llllllll}432 & 1.4014113 & 20.996283 & 0.68374407 & 0.0000 & 2.8127272 & 0.0000 & 1.1994030\end{array}$

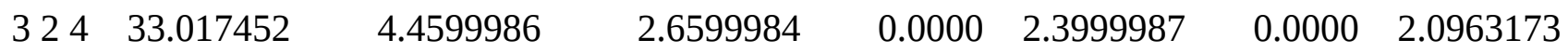

$34246.6804286 .75672486 .5644560 \quad 0.00004 .52938080 .00004 .7479572$

24 ! Nr of torsions ; at1 ; at2 ; at3 ; at4 ; V1 ; V2 ; V3 ; V2(BO) ; vconj ; n.u. ; n.u.

$4444-0.250011 .58220 .1879-4.7057-2.20470 .00000 .0000$

$4441-0.250031 .2596 \quad 0.1709-4.6391-1.90020 .00000 .0000$

$1441-0.177030 .0252 \quad 0.4340-5.0019-2.06970 .00000 .0000$

$4442-0.709822 .2951 \quad 0.0060-2.5000-2.16880 .00000 .0000$

$1442-0.356822 .64720 .6045-4.0088-1.00000 .00000 .0000$

$2442-0.0528 \quad 6.8150 \quad 0.7498-5.0913-1.00000 .00000 .0000$

$44242.000725 .5641-0.0608-2.6456-1.17660 .00000 .0000$

$4421-1.195342 .1545-1.0000-8.0821-1.00000 .00000 .0000$

$1424-0.928434 .39520 .7285-2.5440-2.46410 .00000 .0000$

$1421-2.500079 .69801 .0000-3.5697-2.75010 .00000 .0000$

$4422-0.01795 .0603-0.1894-2.5000-2.03990 .00000 .0000$

$1422-0.558380 .00001 .0000-4.4000-3.00000 .00000 .0000$ 
$2424-2.500076 .0427-0.0141-3.7586-2.90000 .00000 .0000$

$24210.034578 .9586-0.6810-4.1777-3.00000 .00000 .0000$

$2422-2.500066 .35250 .3986-3.0293-3.00000 .00000 .0000$

$42242.5000-0.53321 .0000-3.5096-2.90000 .00000 .0000$

$4221-2.5000 \quad 3.32190 .7180-5.2021-2.93300 .00000 .0000$

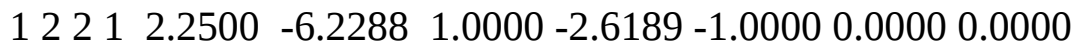

$42220.0531-17.39831 .0000-2.5000-2.15840 .00000 .0000$

$12220.4723-12.4144-1.0000-2.5000-1.00000 .00000 .0000$

$2222-2.5000-25.00001 .0000-2.5000-1.00000 .00000 .0000$

$14430.00000 .00000 .00000 .0000-1.00000 .00000 .0000$

$34240.00000 .00000 .00000 .0000-1.00000 .00000 .0000$

$34210.00000 .00000 .00000 .0000-1.00000 .00000 .0000$

1 ! Nr of hydrogen bonds;at1;at2;at3;Rhb;Dehb;vhb1

$\begin{array}{lllllll}2 & 1 & 2 & 2.1200 & -3.5800 & 1.4500 & 19.5000\end{array}$

\section{Bulk ZnO Thermochemistry}

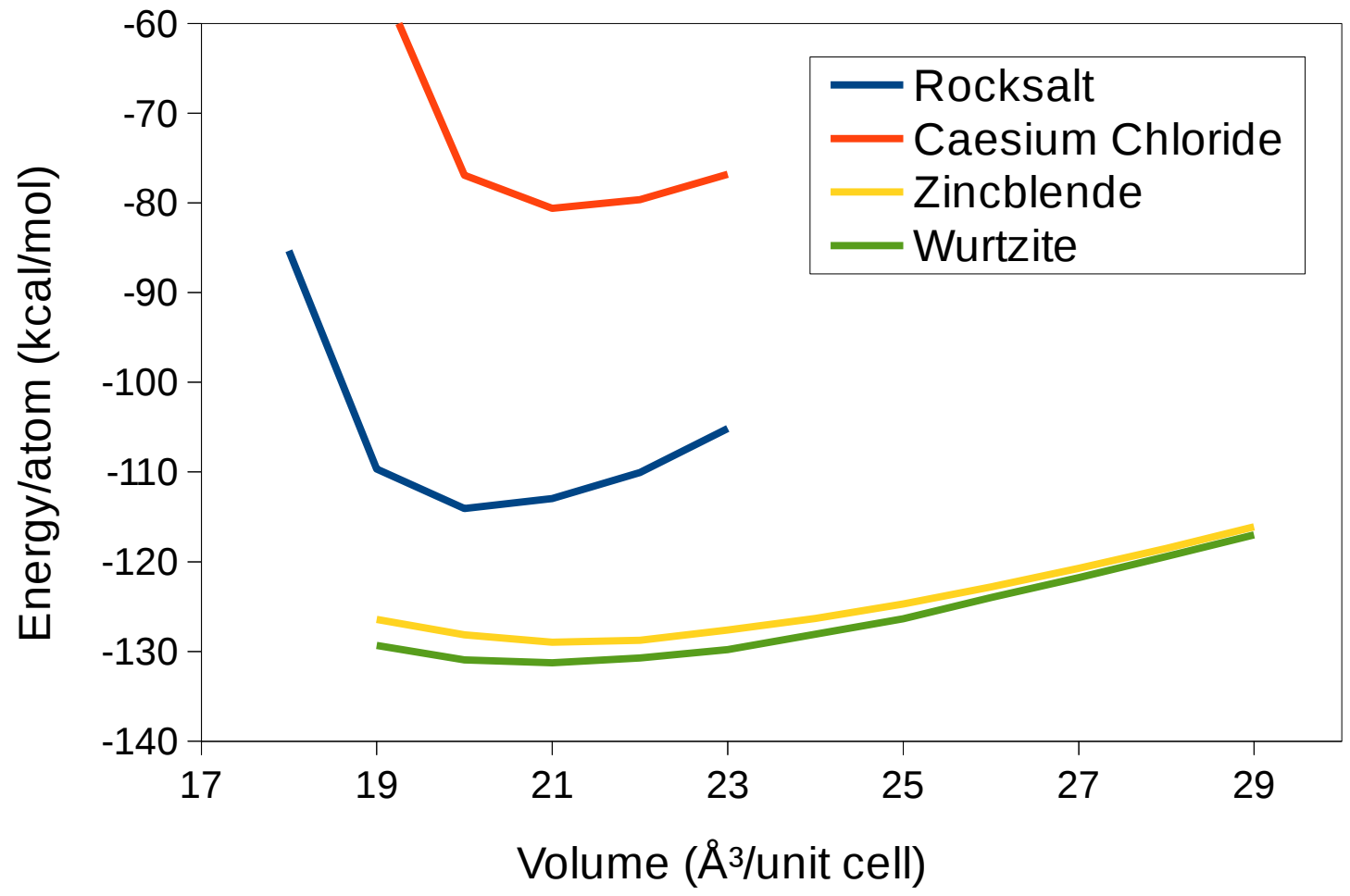

Figure S1: Equation of state for four the wurtzite (green), zincblende (yellow), rocksalt (blue) and caesium chloride (orange) ZnO crystal structures calculated using the ReaxFF parameters developed in this study. We should note that the minimum energies/atom for the wurtzite, zincblende, rocksalt and caesium chloride polymorphs of $\mathrm{ZnO}$ using the parameters from Ref. 1 and the LAMMPS simulation package were -91, -89, -84 and -68 kcal/mol, repsectively. 


\section{Geometry Optimizations}

Below are the bond distance and angle results from geometry optimization calculations carried out at each level of theory (DFT is B3LYP/6-311+G*).

\begin{tabular}{|c|c|c|c|c|c|c|}
\hline & $\begin{array}{c}\text { EZE } \\
\text { DFT }\end{array}$ & $\begin{array}{c}\text { EZE } \\
\text { ReaxFF }\end{array}$ & $\begin{array}{c}\text { EOZE } \\
\text { DFT }\end{array}$ & $\begin{array}{c}\text { EOZE } \\
\text { ReaxFF }\end{array}$ & $\begin{array}{c}\text { EOZOE } \\
\text { DFT }\end{array}$ & $\begin{array}{c}\text { EOZOE } \\
\text { ReaxFF }\end{array}$ \\
\hline Zn-C $(\AA)$ & 1.973 & 1.980 & 1.949 & 1.976 & - & - \\
\hline Zn-O $(\AA)$ & - & - & 1.789 & 1.811 & 1.776 & 1.797 \\
\hline C-Zn-C & $179.89^{\circ}$ & $174.43^{\circ}$ & - & - & - & - \\
\hline Zn-C-C & $115.81^{\circ}$ & $104.85^{\circ}$ & $115.44^{\circ}$ & $104.12^{\circ}$ & - & - \\
\hline C-Zn-O & - & - & $174.93^{\circ}$ & $165.26^{\circ}$ & - & - \\
\hline Zn-O-C & - & - & $131.84^{\circ}$ & $128.85^{\circ}$ & $127.75^{\circ}$ & $132.04^{\circ}$ \\
\hline Zn-C-H & $107.97^{\circ}$ & $109.81^{\circ}$ & $107.47^{\circ}$ & $109.97^{\circ}$ & - & - \\
\hline
\end{tabular}

\section{Reference:}

(1) Raymand, D.; van Duin, A. C. T.; Spångberg, D.; Goddard III, W. A.; Hermansson, K. Water Adsorption on Stepped ZnO Surface from MD Simulation. Surf. Sci. 2010, 604, 741-752. 